\title{
Delocalized and resonant quantum transport in nonlinear generalizations of the kicked rotor model
}

\author{
Laura Rebuzzini, ${ }^{1}$ Sandro Wimberger, ${ }^{2}$ and Roberto Artuso ${ }^{1,3,4}$ \\ ${ }^{1}$ Center for Nonlinear and Complex Systems and Dipartimento di Fisica e Matematica, Università dell'Insubria, \\ Via Valleggio 11, 22100 Como, Italy \\ ${ }^{2}$ Dipartimento di Fisica E. Fermi, Università degli Studi di Pisa, Via Buonarroti 2, 56127 Pisa, Italy \\ ${ }^{3}$ Unità di Como, Istituto Nazionale per la Fisica della Materia, Via Valleggio 11, 22100 Como, Italy \\ ${ }^{4}$ Sezione di Milano, Istituto Nazionale di Fisica Nucleare, Via Celoria 16, 20133 Milano, Italy
}

(Received 12 October 2004; published 24 March 2005)

\begin{abstract}
We analyze the effects of a nonlinear cubic perturbation on the $\delta$-kicked rotor. We consider two different models, in which the nonlinear term acts either in the position or in the momentum representation. We numerically investigate the modifications induced by the nonlinearity in the quantum transport in both localized and resonant regimes and a comparison between the results for the two models is presented. Analyzing the momentum distributions and the increase of the mean square momentum, we find that the quantum resonances asymptotically are very stable with respect to nonlinear perturbation of the rotor's phase evolution. For an intermittent time regime, the nonlinearity even enhances the resonant quantum transport, leading to superballistic motion.
\end{abstract}

DOI: 10.1103/PhysRevE.71.036220

PACS number(s): 05.45.-a, 03.65.Ta, 42.50.Vk

\section{INTRODUCTION}

Recent and ongoing experiments [1] have started to investigate the interplay between the many-body induced selfinteraction in an ultracold atomic gas and an external driving induced by time-dependent optical potentials. The natural setup is to use a Bose-Einstein condensate of alkali-metal atoms, where the nonlinearity parameter can be tuned $[2,3]$, and pulsed optical lattices can be used to impart momentum kicks to the atoms. For such a setup, the Gross-Pitaevskii (GP) equation $[2,3]$ provides a good description of the system, as long as the nonlinearity is not too large, as a study of the stability of linearized excitations around the GP solution has shown $[4,5]$.

In this paper, we analyze the evolution of a cubic nonlinear Schrödinger equation, as present in the GP model, under the perturbation of time-periodic $\delta$ kicks,

$$
i \frac{\partial \psi}{\partial t^{\prime}}=\left[-\frac{1}{2} \frac{\partial^{2}}{\partial \vartheta^{2}}-u|\psi|^{2}+k \cos (\vartheta) \sum_{t=0}^{+\infty} \delta\left(t^{\prime}-t \tau\right)\right] \psi,
$$

where $\vartheta$ and $n=-i \partial / \partial \vartheta$ are the position and the conjugated momentum of the system; we chose units such that $\hbar=1$ and the motion is considered on a ring with periodic boundary conditions $\psi(\vartheta+2 \pi)=\psi(\vartheta)$. The parameters $u$ and $k$ are the nonlinearity coupling and the kicking strength, respectively.

In atom optics experiments, the $\delta$-kicked rotor has been realized with an ensemble of laser-cooled, cold atoms [6], or recently also with an ultracold Bose-Einstein condensate [1], periodically driven with a standing wave of laser light. With the wave number of the laser $k_{L}$, the experimental variables are easily expressed in our units by noting that momentum is usually measured in two photon recoils $\left(2 \hbar k_{L}\right)$, and position in units of the inverse wave number of the standing wave $\left(1 / 2 k_{L}\right)$. Hence, the scaled variables $\vartheta, n$ and the physical ones $\vartheta^{\prime}, p^{\prime}$ are related by $\vartheta=2 k_{L} \vartheta^{\prime}$ and $n=p^{\prime} / 2 k_{L} \hbar$ [7-9].
Our choice of units makes all the relevant quantities (including the ones plotted in figures) dimensionless.

Owing to the periodicity of the $\delta$-kick perturbation, the time $t$ is measured in number of periods $\tau$ and the evolution of the wave function of the system over a time interval $\tau$ is described by the operator $\hat{U}(\tau)$.

A cubic modification of linear Schrödinger dynamics for the $\delta$-kicked rotor may be accomplished by two different models, both considered in the present paper. The correct way to approximate the evolution of the nonlinear Schrödinger equation is to evaluate the nonlinear term in the position representation [10]. In the following we will refer to this first model as model 1 (M1).

Since the Hamiltonian operator presents a time-dependent nonlinear part $u|\psi|^{2} \psi$, in the numerical integration of Eq. (1), the lowest order split method [11] is used and $\hat{U}$ is approximated by the time-ordered product of evolution operators (Trotter-Kato discretization [12]) on small time steps $\tau / L$ (with $L$ integer):

$$
\hat{U}^{(1)}(\tau)=\hat{K} \hat{R}^{(1)}(\tau) \approx e^{-i k \cos (\hat{\vartheta})} \prod_{l=1}^{L} e^{-i \pi \hat{n}^{2} / L 2} e^{i u(\tau / L)|\psi(\hat{\vartheta}, l \tau / L)|^{2}} .
$$

In the numerical simulations, we use a finite Fourier basis of dimension $N$ : the discrete momentum eigenvalues lie on the lattice $p=(m-N / 2)$ and the continuous angle variable is approximated by $\vartheta=(2 \pi / N)(m-1)$ with $m \in \mathbb{Z}, 1 \leqslant m \leqslant N$. Shifting between the coordinate and momentum representations, in the evaluation of the operator $\hat{R}^{(1)}(\tau)$, requires $2 L$ fast Fourier transforms of $N$-dimensional vectors for each kick. In order to get stable numerical results, the splitting interval $\tau / L$ has to be reduced when increasing the nonlinear coupling constant $u$; typical values of the number of steps 
per period range between $L=80000$ and 5000000 . Therefore, investigating either the effects of strong nonlinearities or the dynamics of the system over long times is computationally quite expensive with M1.

A second model [known as the kicked nonlinear rotator and called in the following model 2 (M2)] was introduced in [13]. This model, being a much simpler variant of the kicked rotator (KR) model [14], allows one to perform faster and more efficient numerical computations. by

The evolution operator over one period $\tau$ for M2 is given

$$
\hat{U}^{(2)}(\tau)=\hat{K} \hat{R}^{(2)}(\tau) \approx e^{-i k \cos (\hat{\vartheta})} e^{-i \pi \hat{n}^{2} / 2} e^{i \widetilde{u} \tau\left|\hat{\psi}_{n}\right|^{2}},
$$

where $\hat{\psi}_{n}$ indicates the $n$th component of the wave function of the system in the momentum representation. The change in the phase of each component $\hat{\psi}_{n}$ of the state vector, introduced by the nonlinear term between two kicks, is proportional to the amplitude of the component. In M1 instead, the phase acquired at each instant by the wave function involves all the Fourier components and the phase factor has as the $n$th Fourier component $(u / 2 \pi) \Sigma_{m} \hat{\psi}_{m+n}^{*} \hat{\psi}_{m}$. The two models coincide only if the wave function of the system is a plane wave of fixed momentum; in this case, the relation between the nonlinear coupling constants in M1 and M2 is $\widetilde{u}=u / 2 \pi$.

Both models M1 and M2 are nonlinear generalizations of the KR and reduce to the KR in the limit $u \rightarrow 0$. Depending on the commensurability of the period $\tau$ of the $\delta$-kick perturbation with $4 \pi$, the KR displays different regimes, deeply studied both theoretically [14-16] and experimentally $[6,8]$. The quantum resonant regime, corresponding to values of $\tau$ being rational multiples of $4 \pi$, is characterized by a ballistic transport: the mean energy of the system grows according to a parabolic law $[15,17]$. For generic irrational values of $\tau$, the average energy grows linearly in time only within a characteristic time (break time), after which dynamical localization sets in and the diffusion is suppressed [16].

In this paper, we analyze in detail how the presence of the nonlinearity affects the general properties of transport in regimes that correspond to the localized (Sec. II, which is essentially a warm up exercise in which the results of [13] are reproduced and additional numerical results about prefactor scaling are presented) and resonant (Sec. III) ones of the KR. We focus our attention on the growth exponent of the mean energy and on how the diffusion coefficient or the rate of ballistic transport depends on the strength of the nonlinear coupling constant $u$. While in Secs. II and III we are dealing with the evolution of an initial state with fixed momentum, chosen at $n=0$, i.e., $\hat{\psi}_{n}(0)=\delta(0)$, Sec. IV is devoted to the effects of a finite spread of initial conditions as strongly suggested by state-of-the-art experiments using ultracold atoms $[1,19]$.

The quantity we typically compute is the width of the momentum distribution of the system $\left\langle p^{2}(t)\right\rangle=\Sigma_{n=-\infty}^{+\infty} n^{2}\left|\hat{\psi}_{n}\right|^{2}$, which gives the spreading of the wave packet over unperturbed levels or equivalently-apart from a constant factor 2 - the expectation value of the energy. The time-averaged spreading $P^{2}(T)=(1 / T) \Sigma_{t=1}^{T}\left\langle p^{2}(t)\right\rangle$ of the second moment has been frequently used (see, e.g., [18]), as it preserves the exponent of the power-law growth, while smoothing out oscillations.

As pointed out in [13] the nonlinear shift is essential in determining dynamical features (providing for instance a mechanism for delocalization of the generic, irrational case), but when we deal with delocalized states it is typically quite small (e.g., for M2 the shift is proportional to $\hat{u} / \Delta n$, where $\Delta n$ is the width of the distribution over unperturbed states). So in general we may expect that, for moderate nonlinearities, the precise form of the shift does not alter in an essential way the nature of the asymptotic motion.

\section{LOCALIZED REGIME}

In this section, we consider the regime where the value of the period of the $\delta$-kick perturbation is incommensurate with $4 \pi$. For better comparison we fix $\tau=1$ for all numerical computations shown in the following. The corresponding system in the $u \rightarrow 0$ limit is characterized by the phenomenon of dynamical localization [16] caused by quantum interference effects. Previous theoretical predictions and numerical simulations $[13,20]$ indicate that, above a critical border $u_{c} \sim 2 \pi$ for the nonlinear coupling constant, dynamical localization is destroyed. The delocalization takes place in the form of anomalous subdiffusion with an exponent of 2/5: in [13] an asymptotic law $\left\langle p^{2}(t)\right\rangle \sim c(u) t^{2 / 5}$ [where $c(u) \sim u^{4 / 5}$ ] is predicted for both models; this is confirmed for both models by the data reported in Fig. 1. In Fig. 1(a) a bilogarithmic plot of the time-averaged second moment vs time is shown for increasing values of the nonlinear coupling constant $u$. In spite of large oscillations, both models fit the predicted asymptotic behavior with a power-law exponent equal to $2 / 5$. For nonlinear coupling larger than the critical border $u_{c}$, marked by the vertical line, the dependence of $c(u)$ vs $u$ is confirmed for both models by Fig. $1(b) . \log _{10}[c(u)]$ is obtained by a oneparameter linear fitting of the logarithm of the second moment, once an anomalous diffusion exponent equal to $2 / 5$ is assumed.

The results obtained by calculating the evolution of the system with either M1 or M2, starting from the same state $\hat{\psi}_{n}(0)=\delta(0)$ and parameters, appear to be different on short time scales; nevertheless the two models share the same asymptotic behavior of the time evolution of the second moment and of the dependence of $c(u)$ vs $u$. The effect of the nonlinearity is the same for both models only at $t=1$, because of the common initial state. As explained in the Introduction, the way nonlinearity acts on the wave function is essentially different for M1 and M2, at least before the state becomes delocalized: so deviations are qualitatively expected for intermediate times, while we expect a closer analogy in the models' behavior in the asymptotic regime. Actually the close behavior exhibited by both models in Fig. 1 after a few time steps extends to more general features than the second moment: in Fig. 2 we provide a comparison between full distributions over momentum states for $u=10$.

We remark that nonlinearity-induced delocalization has recently been explored also in studying survival probability 

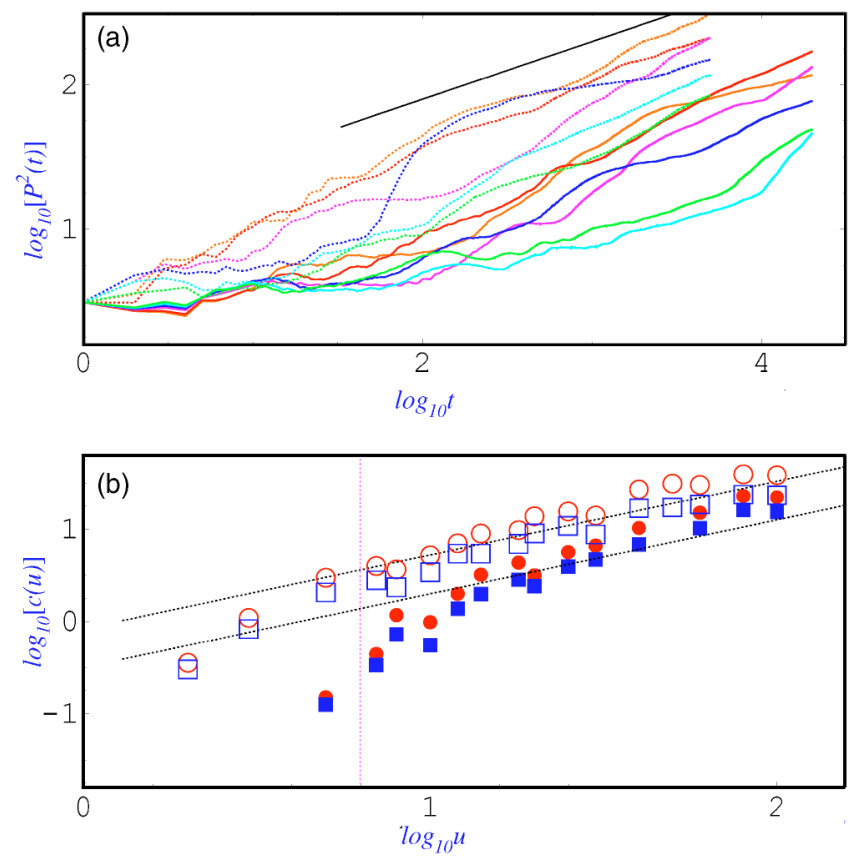

FIG. 1. (Color online) (a) Bilogarthmic plot of the timeaveraged second moment vs time in the localized regime. Time is measured in number of periods. The dashed and full lines refer to M2 with $N=2^{17}$ and M1, with $N=2^{9}$ and $L=80000$, respectively. Values of $u=8,10,12,14,16,20$ are considered; generally higher nonlinearity values yield bigger spreading. The dashed line has the theoretically predicted slope $2 / 5$. The values of the parameters are $\tau=1$ and $k=2.5$; the initial state is $\hat{\psi}_{n}(0)=\delta(0)$. (b) The logarithm of the coefficient of the sub-diffusion as a function of $\log _{10}(u)$, for the second moment (circles) and its time average (squares). Empty and full symbols refer, respectively, to M1 and M2. The dashed lines show the predicted dependence $\sim u^{4 / 5}$.

on a finite momentum sample [21]: while the authors use M2 to cope with computational difficulties, our findings suggest that their results are probably relevant for true nonlinear Schrödinger equation dynamics too.

\section{RESONANT REGIME}

In this section, we examine in detail the response of the system to nonlinear perturbation in the resonant regime of the $\mathrm{KR}$ ( $\tau=4 \pi r / q$ with $r, q$ relatively prime integers), characterized by a parabolic growth in time of the variance of the momentum distribution $[15,17]$. The value of $\tau$ is chosen equal to $4 \pi$, corresponding to the first fundamental quantum resonance of the KR.

In Fig. 3(a) a bilogarithmic plot of the time-averaged second moment of the momentum distribution for different values of the nonlinearity is shown. The nonlinear coupling constant $u$ varies from 1 to 400. As already noticed [20], the resonant behavior survives even in the presence of nonlinearity, although generically the spreading is slowed with respect to the linear case. On asymptotically long time scales, resonant growth with a quadratic exponent is reached even for strong nonlinear perturbations, though we observe that the time needed to reach the asymptotic regime grows with
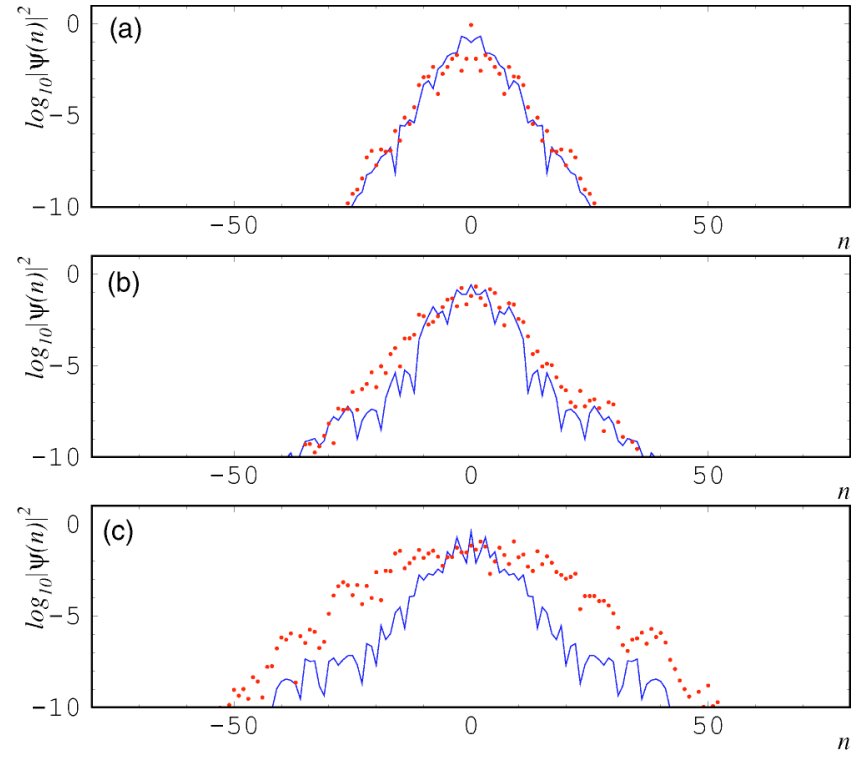

FIG. 2. Comparison between the momentum distributions for M1, circles, and M2, full line, after $t=10$ (a), 100 (b), and 1000 (c) kicks. The parameters are the same as in Fig. 1; the nonlinear coupling is fixed to the value $u=10$.

$u$. The results in Fig. 3(a) obtained from M2, shown in full lines, allow us now a more detailed analysis of the behavior of the system at quite long times. It can also be seen in Fig. 3(a) that the time evolution of the second moment of M1, shown with circle symbols, approaches the same asymptotic growth, even if some differences between the two models appear especially for large nonlinearity $(u \gtrsim 50)$.

The persistence of the resonant behavior in the presence of nonlinearity can be explained intuitively as follows. In the linear (i.e., $u=0$ ) resonant case the width of the momentum distribution increases linearly in time. Therefore, from the normalization condition, the probability amplitude to find the system in a momentum eigenstate $n$ decays as $\left|\hat{\psi}_{n}\right|^{2} \sim 1 / \Delta n$ $\sim 1 /(\pi k t)[7,13]$. The nonlinear phase shift $\tau u\left|\hat{\psi}_{n}\right|^{2}$ decreases with the same rate and its effects become irrelevant on long time scales, i.e., $t \gg(u / \pi k) \tau$.

Nevertheless, the nonlinearity affects the evolution of the second moment on smaller time scales $t \lesssim(u / \pi k) \tau$ and introduces a $u$ dependence in the prefactor of the parabolic growth law $\left\langle p^{2}(t)\right\rangle \sim a(u) t^{2}$. Increasing nonlinear coupling manifests in a slower quadratic growth. In Fig. 3(b) the function $\Delta^{(\bar{u})}(t)=\left[\left\langle p^{2}(t)\right\rangle^{(\widetilde{u})}-\left\langle p^{2}(t)\right\rangle^{(0)}\right] / t^{2}$ is plotted for different values of the nonlinearity. Increasing asymptotic absolute values of $\Delta\left(t_{\max }\right)$ give an estimate of the modifications in the transport induced by the nonlinearity. The coefficients $a_{\Delta}$, calculated from the function $\Delta\left(t=t_{\max }\right)$ with $t_{\max }=20000$, are shown in the inset of Fig. 4.

A detailed analysis of the dependence of the coefficient $a(u)$ of $\left\langle p^{2}(t)\right\rangle$ is presented in Fig. 4. The numerical calculation of $\log _{10}[a(u)]$ is obtained by a one-parameter linear fitting of the logarithm of the second moment vs the logarithm of time with a straight line of fixed slope 2 . The fitting is performed on a time interval $\Delta t=200$; this rather small time interval was chosen in order to make a comparison between 

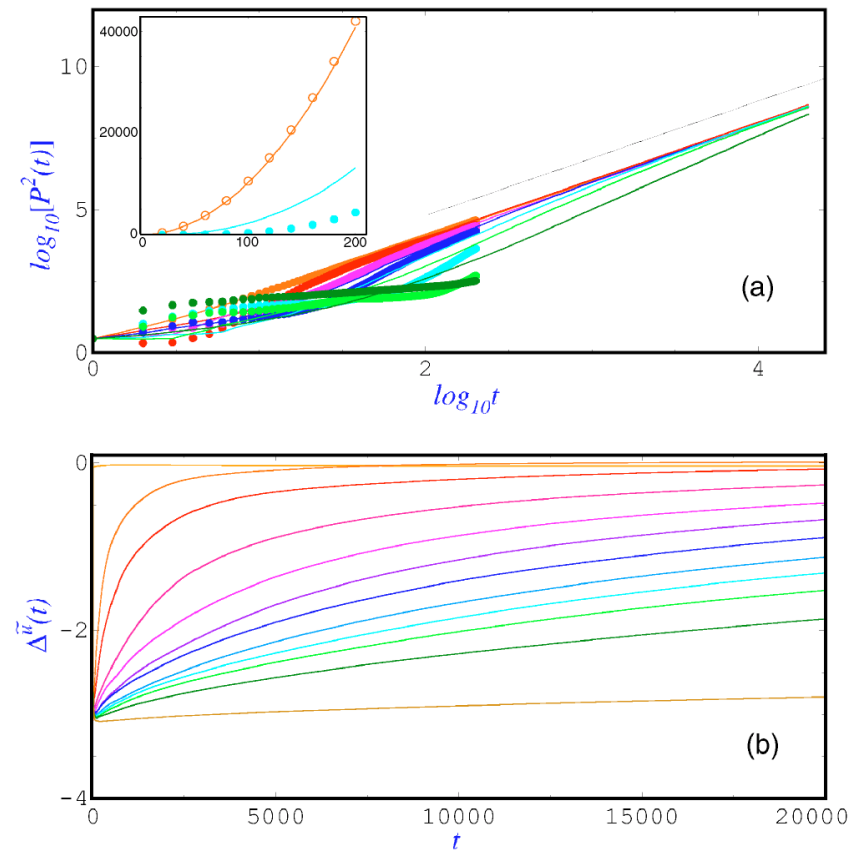

FIG. 3. (Color online) (a) Resonant growth of the time-averaged second moment vs time in the presence of nonlinearity, for $\tau=4 \pi$ and $k=2.5$. The initial momentum distribution is $\hat{\psi}_{n}(0)=\delta(0)$. The symbols and the full lines refer to M2 with $N=2^{17}$ and M1 with $N=2^{10}$ and $L=5000000$, respectively. The straight black line shows the resonant asymptotic behavior $t^{2}$. The values of the nonlinear parameter are $u=1,5,10,20,50,100,400$. The inset is a magnification for $u=1$ and 50 (lower part). A slight deviation between the two models can be seen for $u=50$. (b) The function $\Delta^{(\widetilde{u})}(t)$ vs time for M2. Starting from above in the low $t<5000$ region, the values of the nonlinear parameter are $\tilde{u}$ $=0.1,5,10,20,30,40,50,60,70,80,100,400$.

the results from both models (empty and full symbols refer to $\mathrm{M} 1$ and M2, respectively). The accordance between the two models is satisfying up to $u \lesssim 50$. For $u>50$ the lowest order split method [11] to evaluate the Floquet operator in M1 becomes less stable and the numerical errors around the borders of the finite basis propagate faster.

Numerical data are compatible with an algebraic law $a(u) \simeq k^{2} /\left[2(1+u / c)^{\gamma}\right]$, where $\gamma$ is $4 / 5$ and $c$ is a constant of the order of 10 (for the time-averaged moment the constant $k^{2} / 2$ is substituted by $\left.k^{2} / 6\right)$. This law has the required asymptotic behavior for $u \rightarrow 0$ : in this limit $a(u)$ tends to the well-known value of the coefficient of the resonant KR, i.e., $a(0)=k^{2} / 2$. In Fig. 4 the values of $a(0)$ are marked by arrows. For large values of $u, a(u)$ decreases for increasing nonlinearity with the inverse power law $\sim u^{-4 / 5}$. At the moment we have no explanation for the minimum observed in the intermediate region $\left(\log _{10} u \sim 0.85\right)$.

Up to now, we discussed only the case of attractive interactions, i.e., $u>0$. It turns out that the fundamental quantum resonance at $\tau=4 \pi$ is insensitive to the sign of the nonlinearity as can be seen in the inset of Fig. 5. The same is true for the momentum distributions, which are not presented here. On the other hand, the next order resonance at $\tau=3 \pi$ is sensitive to the sign of $u$. For $u<0$ in Fig. 6(b), the momen-

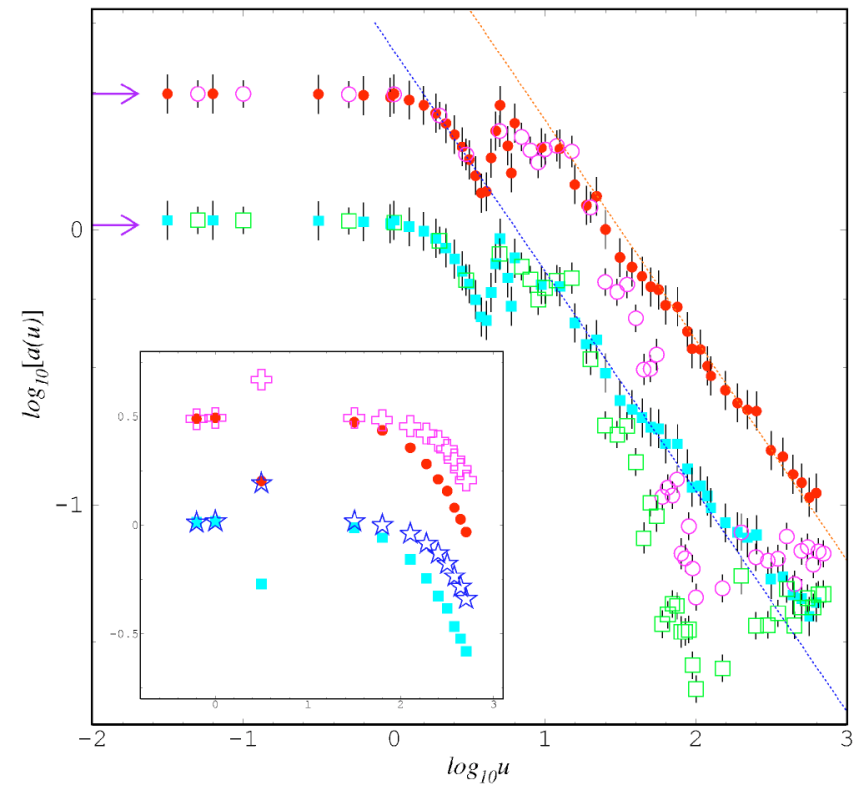

FIG. 4. (Color online) Bilogarithmic plot of the coefficient $a(u)$ of the quadratic growth of the second moment (circles) and its time average (squares) as a function of the nonlinear parameter $u$. Empty and full symbols refer to M1 and M2, respectively. The dashed lines show the algebraic behavior of $a(u)$ for large $u$ with an exponent equal to $-4 / 5$. Notice that for $u \leqslant 1$ the coefficients approach the theoretical values of the KR model, marked by arrows. In the inset the empty symbols refer to $a_{\Delta}$ calculated using the function $\Delta(t$ $=t_{\max }$ ) with $t_{\max }=20000$.

tum distribution is slightly different from $u>0$. Asymptotically, however, the same ballistic growth of the mean square momentum is obtained. This means that the details of the effect of nonlinearity depend on the resonance type as far as the sign of $u$ is concerned. This originates from the fact that while at the fundamental quantum resonances $\tau=4 \pi m$ ( $m$ $>0$ integer) the free evolution phase in the linear rotor is

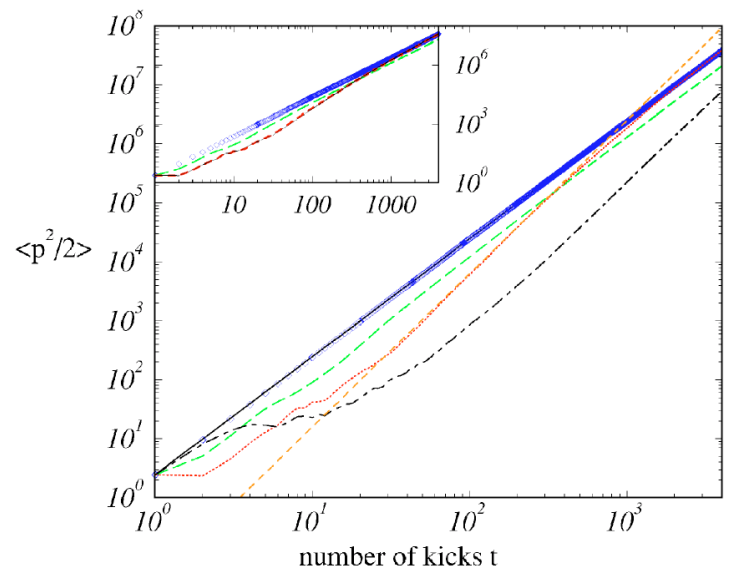

FIG. 5. Kinetic energy at the fundamental quantum resonance $\tau=4 \pi, k=\pi, p_{\text {initial }}=0$, and nonlinearities $\tilde{u}=0$ (solid), -0.2 (diamonds), -1 (dashed), -10 (dotted), and -100 (dash-dotted). The short dashed line shows a superballistic increase proportional to $t^{2.6}$ for the case $\tilde{u}=-10$. The inset presents the results for $\tilde{u}=0.2,1,10$, and the case of $\tilde{u}=-10$ (thin solid line) for better comparison. 

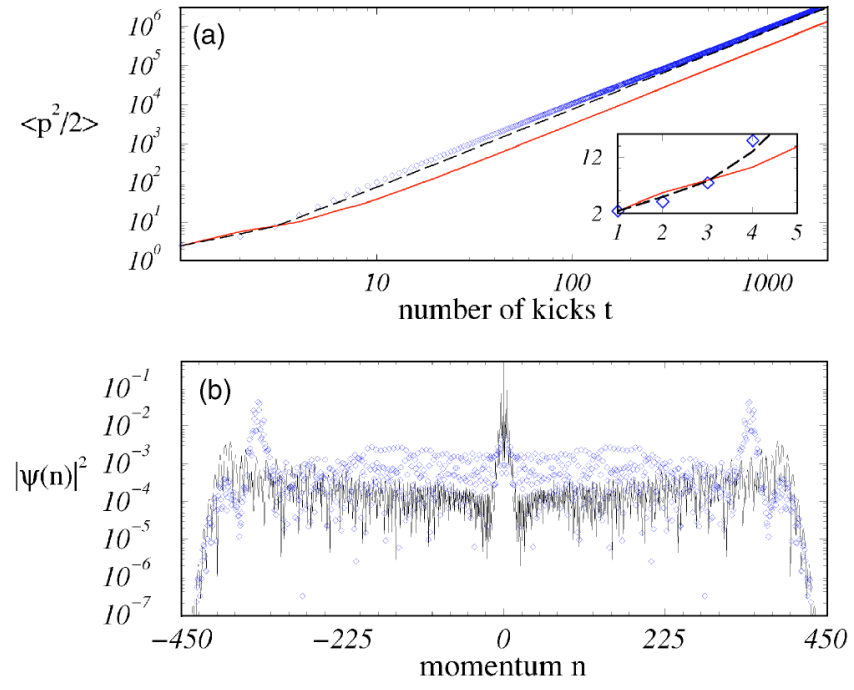

FIG. 6. (a) Kinetic energy as a function of the number of kicks, and (b) the corresponding momentum distributions after 200 kicks, for the quantum resonance $\tau=3 \pi$. Same parameters as in Fig. 5, apart from the nonlinearity, which is $\tilde{u}=-0.2$ (diamonds) and 0.2 [(a) dotted, (b) solid]. In (a) we show also data for $u=0$ (dashed) for comparison, and the inset illustrates the opposite effect of the second and fourth kicks (the rephasing in momentum space occurs for the $u=0$ case only every second kick at $\tau=3 \pi$ ) depending on the sign of $\tilde{u}$.

exactly 1, at higher order resonances there is a nontrivial phase evolution between two successive kicks. The inset of Fig. 6(a) highlights that $\left\langle p^{2}\right\rangle / 2$ either decreases or increases with respect to the case $u=0$ at the second and fourth kicks. This is related to the fact that at $\tau=3 \pi$ the rephasing in momentum space occurs only every second kick, not between two successsive kicks as at $\tau=4 \pi$.

In Figs. 5 and 6 the time scales relevant for experiments $(t \lesssim 500)$ are investigated (results refer to M2). In Fig. 5, we generically observe three regimes. (i) There is an initial stage, where the mean square momentum increases much more slowly than in the case $u=0$. This stage is followed by stage (ii) where the increase can be faster than ballistic, and the mean square momentum can even be larger for larger nonlinearity (see $|\tilde{u}|=10$ as compared to $|\tilde{u}|=1$ in Fig. 5).

The observed superballistic growth of the second moment of the momentum distribution is quite surprising, in particular, having in mind that such a growth is forbidden in the usual KR (i.e., $u=0$ ) [22]. The results in Fig. 5 are reminiscent of the observed superballistic spreading in onedimensional tight-binding models [23]; however, here the superballistic behavior is caused by the nonlinear term in the time evolution, in contrast to the linear Hamiltonian models in [23]. In terms of the model studied in [23], the nonlinearity $u$ would act as a finite size trapping region (cf. also [21]), outside of which the motion is ballistic (we already showed how nonlinearity does not essentially modify high $n$ components).

The final stage (iii) we call the asymptotic regime, because there the growth exponent approaches the one for vanishing nonlinearity (only for $\tilde{u}=-100$ is this stage not yet reached in Fig. 5).

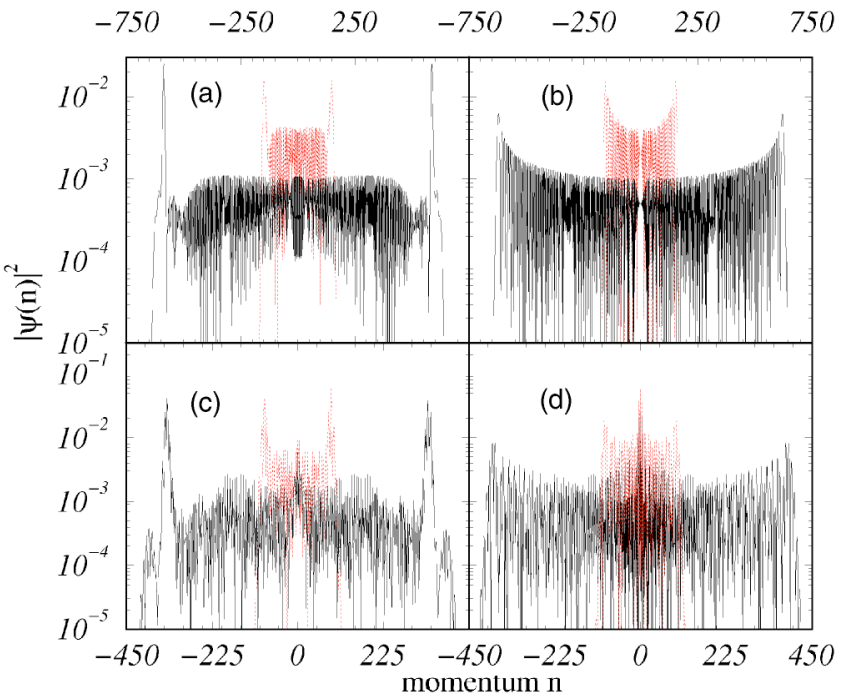

FIG. 7. Momentum distributions for zero initial momentum, $k$ $=\pi, \tilde{u}=-0.2$ (a), (c) and $u=0$ (b), (d), and $\tau=4 \pi$ (a), (b), $\tau=3 \pi$ (c),(d). Note the stable peaks at the largest momenta in the case with small nonlinearity. The distribution are shown after 50 (dotted) and 200 (solid line) kicks in each panel.

In Fig. 6(b) we notice distinct peaks close to the very edge of the momentum distribution for $\tilde{u}=0.2$. Such peaks have been found for sufficiently large kicking strength $k \gtrsim 2.5$ and corresponding $|\tilde{u}(k)|=0.2, \ldots, 2$, and it turns out than they can be up to one order of magnitude higher than the maximum of the momentum distribution for the linear KR at the resonances $\tau=4 \pi$ and $3 \pi$. Figure 7 compares the momentum distribution $\left|\hat{\psi}_{n}\right|^{2}$ at the fundamental quantum resonance $\tau$ $=4 \pi$, and at the resonance $\tau=3 \pi$ for small nonlinearity $\tilde{u}$ $=-0.2$ with the case of the linear KR. The distributions are shown after 50 and 200 kicks, respectively, to stress their evolution in time. For both resonances, we observe a very interesting feature, namely, the small nonlinearity sharpens the edge peaks, which move ballistically, i.e., with a speed that is proportional to the number of kicks $t$ [we recall that when $u=0$ the distribution is characterized by a largest momentum component also moving according to a linear law $\left.n_{\text {max }}(t) \simeq k t \pi / 2[7]\right]$. The peaks are more pronounced than in the linear case, and are remarkably stable, i.e., their height decreases very slowly with increasing number of kicks in Fig. 7(c), or even increases initially as in Fig. 7(a). While we focused our discussion on the model M2, the structure of the probability distribution is quite similar for M1 (see Fig. 8).

The intermediate time scaling properties look in this case more complex than in the kicked rotator dynamics in the presence of sticking accelerator modes, where the same exponent appears both in the classical and in the quantum cases (where a new modulation appears). Work is in progress to see whether there exist classical mappings that reproduce the peak dynamics we observe in the intermediate time quantum behavior [24].

\section{MOMENTUM DIFFUSION IN THE RESONANT REGIME}

All the above results have been obtained for an initial state in the form of a plane wave of null momentum. In a 

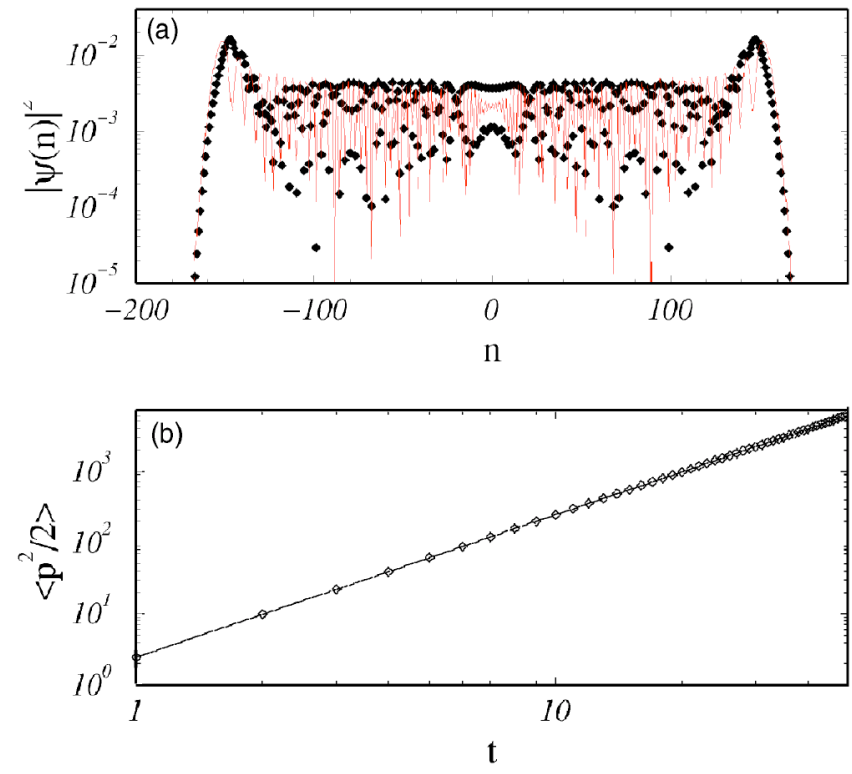

FIG. 8. (a) Momentum distribution after $t=50$ kicks, and (b) mean square momentum for $p_{\text {initial }}=0, u=-0.2, \tau=4 \pi$, and $k=\pi$. Shown are the evolutions induced by M2 (diamonds) and by M1 (full lines).

typical experiment, one can create a Bose condensate with an initial spread of momentum which is much less than two photon recoils (which can be imparted as momentum kicks to the atoms by the kicking laser). We ask ourselves what happens if such a spread is taken into account. The momentum variable $p$, varying on a discrete lattice in the case of a single rotor, becomes a continuous variable. For the linear $\mathrm{KR}$, the eigenvalues of $\hat{p}$ can be written, distinguishing the integer and fractional parts (quasimomentum), as $p=[p]$ $+\{p\}=n+\beta$. Owing to the conservation of quasimomentum $\beta$, the system dynamics can be decomposed into a bundle of rotors [26] (called in the following $\beta$ rotors), each parametrized by a value of the quasimomentum, evolving incoherently with operators with the same functional form as Eqs.

(2) and (3), in which $\hat{n}$ is substituted by $\hat{n}+\hat{\beta}$. Such a decomposition is not easily accomplished when we introduce a nonlinear term in the dynamics. The general task we have to face becomes the study of a nonlinear evolution equation with periodic coefficients. This is a quite a complex problem that cannot be tackled in full generality, even though different approximation schemes have been proposed, e.g., by mapping the problem into a discrete lattice, which turns out to be useful if the wave function is expanded in a suitable set of localized functions related to the linear problem [27]. We generalize M2 in such a way that its linear limit is the evolution operator corresponding to a quasimomentum $\beta$ (as formerly specified), and assume that each nonlinear $\beta$ rotor evolves independently. In this way we study the influence of nonlinearity on realistic initial conditions in a highly simplified way, by means of a generalized M2 model: further work is obviously needed to check whether our findings extend to a full GP dynamics [28].

The quantum resonance phenomenon in the $\mathrm{KR}$ is strongly sensitive to the values of the parameters of the sys- tem. The linear KR rotor $(u=0)$ exhibits the quantum resonance only for a finite set of quasimomenta, i.e., $\beta=\beta^{R}$ $=m / 2 p$ with $m<2 p[7,15,26]$. A slight deviation of the quasimomentum from $\beta^{R}$ changes the evolution of the system completely. For values of $\beta \neq \beta^{R}$, after a transient regime, the suppression of the resonant growth of the energy of the linear $\beta$ rotor through dynamical localization occurs; at fixed time $t$, only quasimomenta within an interval $\sim 1 / t$ of $\beta^{R}$ mimic the ballistic behavior proportional to $t^{2}$ and a rough estimate of the time up to which the quadratic growth of the $\beta$-rotor energy persists is $\bar{t} \sim 1 / \Delta \beta$, where $\Delta \beta=\left|\beta-\beta^{R}\right|$ $[7,25]$.

In the following, we investigate the mean square momentum distribution of the generalized M2 model, first (a) for a single $\beta$ rotor with fixed quasimomentum, and then (b) for an incoherent ensemble of $\beta$ rotors whose initial state in momentum space is a Gaussian distribution with zero mean and rms spreading $\Delta \beta=\sigma=0.01$. We choose $\tau=4 \pi$, and the resonance condition is then met for $\beta^{R}=0$ and $\beta^{R}=1 / 2$. The case with $\beta^{R}=0$ fixed was considered in Sec. III. As in the localized regime, considered in Sec. II, the introduction of the nonlinearity causes a delocalization in the system with a nonresonant value of the quasimomentum $\left(\beta \neq \beta^{R}\right)$. For small nonlinearities, the appearance of an anomalous asymptotic diffusion with an exponent of $2 / 5$, after the initial ballistic behavior, is confirmed by data of Fig. 9(a) for a $\beta$ rotor with $\beta \approx 0.009$. On the contrary, greater nonlinear couplings $(u$ $\geqslant 50$ ) introduce an excitation of diffusive type, starting from the first kicks. In Fig. 9(b) the quasimomentum of the $\beta$ rotor is varied and $u$ is kept fixed. The arrows mark the times $\bar{t}$, depending on the value of $\beta$, approximately bounding the region of the ballistic growth.

We then consider the dynamics of an incoherent ensemble of $\beta$ rotors. The mean square displacement of the distribution is $\left\langle p^{2}(t)\right\rangle_{\beta}=\int d \beta\left\langle p_{\beta}^{2}(t)\right\rangle$. The average over $\beta$ has been calculated using 5000 quasimomenta. In Fig. 10 the time evolution of the averaged second moment of the initially Gaussian wave packet is shown for M2. The behavior in the corresponding linear case of the KR is theoretically known (see Appendix A of [26]): for $u=0$, the kinetic energy of the system increases diffusively in time with a coefficient proportional to $k^{2} / 4$, and dependent on the initial distribution of quasimomenta [7]. The presence of the nonlinearity manifests itself in a faster than linear growth, at least on short time intervals. After this transient regime, the asymptotic growth is expected to become approximately linear. The black straight line is drawn for better comparison. At fixed time $t$ and assuming a uniform distribution of the quasimomenta, the resonant rotors whose quasimomenta lie within the interval $\Delta \beta$, enter in the average of $\left\langle p^{2}\right\rangle_{\beta}$ with a contribution of $\sim t^{\alpha}$ and a weight $w \sim 1 / t$, while the nonresonant rotors give a contribution of $(1-w) t^{\gamma}$. The exponents of the transport in the limit $t \rightarrow+\infty$ reach the values $\alpha(\infty)=2$ and $2 / 5 \leqslant \gamma \leqslant 1$. Therefore, asymptotically in time, the global transport exponent reaches the value 1. In the inset of Fig. 10 the exponents of the algebraic growth of the second moment are plotted as a function of the nonlinear coupling constant $\tilde{u}$. The fitting time interval is 1000 kicks. Full and open circles refer to 5000 and 500 quasimomenta of the initial Gaussian 

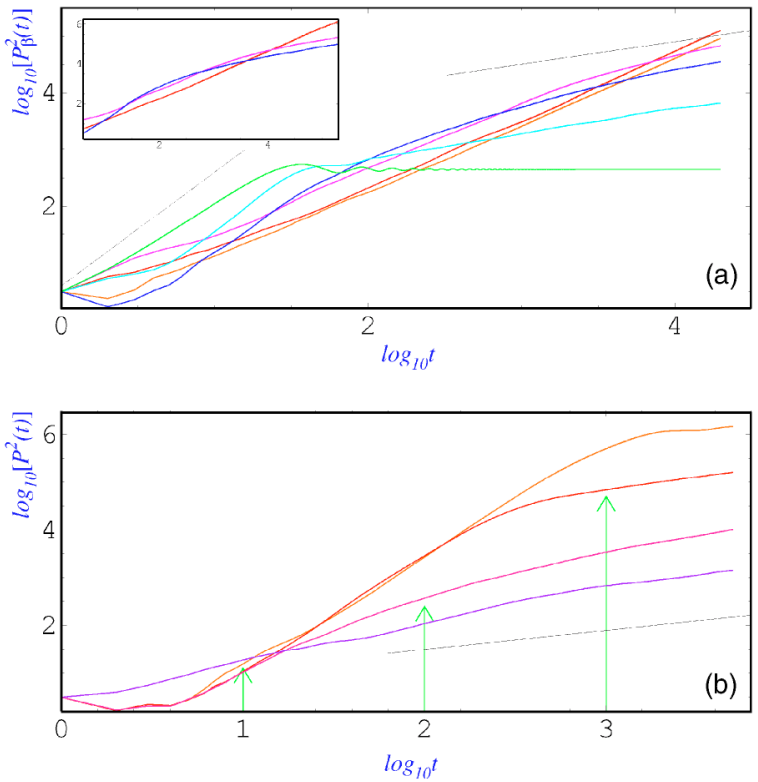

FIG. 9. (Color online) (a) Bilogarithmic plot of the second moment of a single $\beta$ rotor with a fixed quasimomentum for increasing nonlinear coupling (from below, referring to high $t$ values, $\tilde{u}$ $=0,1,5,10,50,100)$. The two lines have slope 2 and $2 / 5$. Note that in the KR case $(u=0)$, the localization occurs for time $t \gtrsim 1 / \beta$. The parameters are $\tau=4 \pi, k=2.5$, and $N=2^{17}$. The initial state is an eigenfunction of the momentum with $n=0$ and $\beta=0.00946556$. In the inset the calculations are prolonged ten times. The unbounded growth for $\tilde{u}=50,100$ can be clearly seen. (b) The same as (a) with $u$ fixed $(\tilde{u}=5)$ and variable $\beta$ (from above, referring to high $t$ values, $\beta=0.0001,0.001,0.01$, and 0.1$)$. The arrows mark the times $1 / \beta$.

distribution: a slight rise in the exponents can be noted on increasing the number of quasimomenta, because a greater number of them approach the value $\beta^{R}=0$, yielding the quadratic growth of the $\beta$-rotor energy. Note that the exponent approches faster the value 1 for a uniform initial distribution of quasimomenta (stars), confirming the previous argument.

Figure 11 presents a closer look at the dynamics on a shorter time scale: the results refer to the case in which we found stable momentum peaks in Fig. 7. Part of the peak is still preserved for the used spread $\Delta \beta \simeq 0.01$, which can be realized in state-of-the-art experiments $[1,19]$. After about 15 kicks, more weight lies, however, now in the center of the distribution made up of rotors which do not exactly satisfy the rephasing condition due to nonzero quasimomenta. Also the increase of the mean square momentum, which is averaged incoherently over all the independently evolved initial conditions, is then not any more quadratic but closer to linear (see inset in Fig. 11), as was found in the case of a uniform initial distribution of quasimomenta for the $u=0$ case $[7,25]$. The mean square momentum still increases much faster than for nonresonant values of the kicking period $\tau$, where dynamical localization occurs. The latter may be destroyed by the nonlinearity but the above observed growth of $\left\langle p^{2}\right\rangle / 2$ $\propto t^{2 / 5}$ (cf. Sec. II) is much slower than linear. On short time scales thus quantum resonance is very robust with respect to nonlinear perturbations. If our incoherent superposition

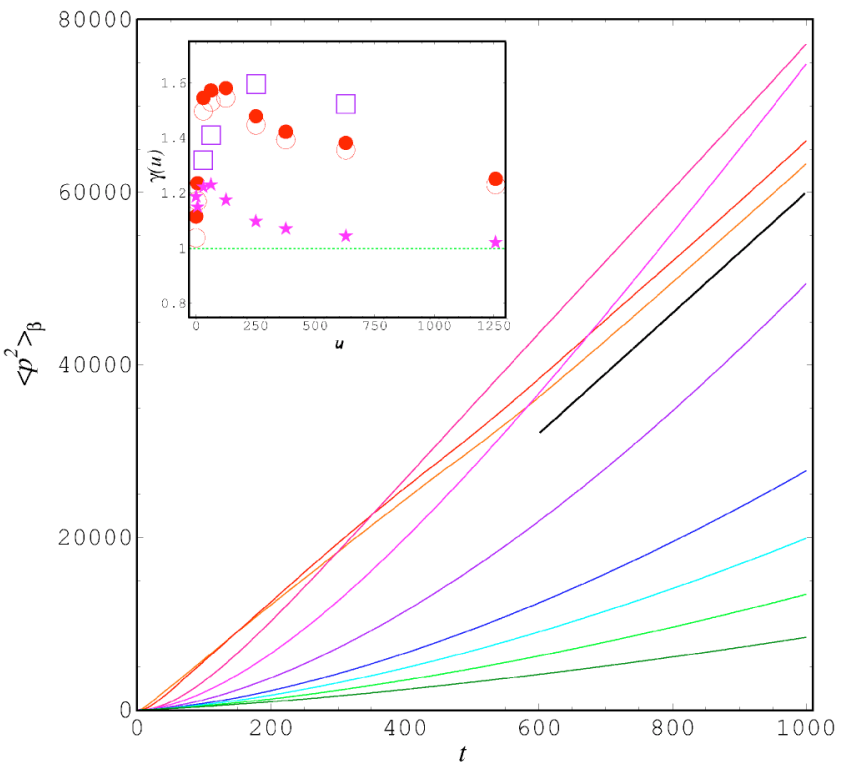

FIG. 10. (Color online) Average over 5000 quasimomenta of the second moment of the distribution vs time for M2; $\tau=4 \pi$ and $k$ $=2.5$. Starting from below (referring to high $t$ values) $\tilde{u}$ $=200,100,60,40,20,0,1,5,10$; the initial wave packet in Fourier space is a Gaussian distribution centered at $n=0$ with $\mathrm{rms} \sigma=0.01$. The inset shows the power-law exponents of the second moment as a function of $\tilde{u}$. The fitting is performed on time intervals $\Delta t$ $=1000$ (circles) and 6000 (squares). Open symbols refer to 500 quasimomenta. Stars refer to a uniform distribution of quasimomenta.

model is correct after some initial stage, the ballistic motion should cease but the dynamics will show the influence of the ballistic quantum resonant transport.

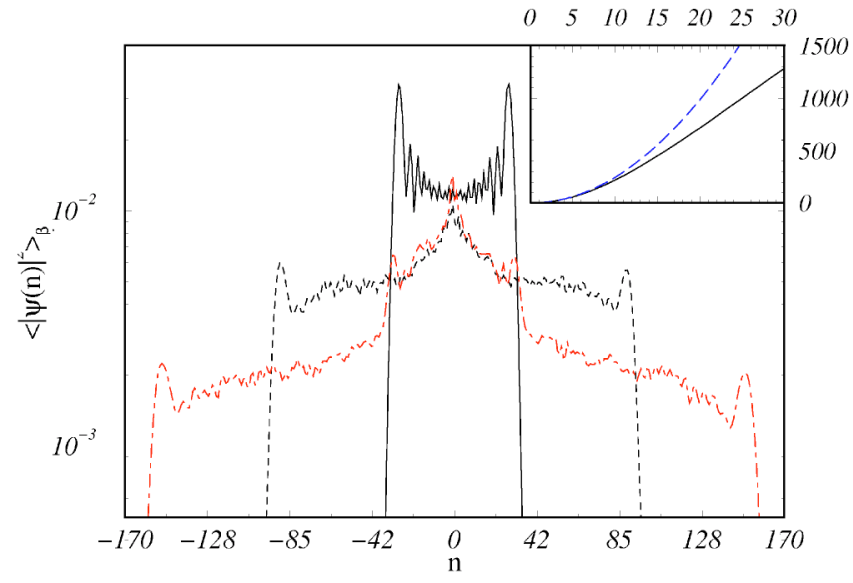

FIG. 11. Momentum distribution after $t=10$ (solid), 30 (dashed), and 50 kicks (dash-dotted), for the same parameters as in Fig. 7(a), but incoherently averaged over independently evolved initial conditions (Gaussian initial momentum distribution with rms $\sigma=0.01$ centred around $n=0)$. The inset shows the corresponding $\left\langle p^{2}\right\rangle_{\beta} / 2$ as a function of the number of kicks for $\tilde{u}=-0.2$ (solid) and $u=0$ (dashed). 


\section{CONCLUSIONS}

In summary, we have numerically analyzed in great detail the quantum transport occurring in two nonlinear generalizations of the famous $\delta$-kicked rotor model, with a cubic nonlinearity as present in the Gross-Pitaevskii equation. We confirm previous results in the regime of localized transport, and show the validity of the predictions of [13] for a wide range of nonlinear coupling strengths. In addition, we found that the quantum resonances of the kicked rotor are very stable with respect to the nonlinear phase perturbation, which loses its effect in the asymptotic limit of large interaction times with periodic driving. Surprising phenomena like pronounced peaks in the momentum distributions at quantum resonance and superballistic intermittent growth of the mean square momentum have been found. Both phenomena are caused by cubic nonlinearity in the evolution, which shows that the analyzed models bear a rich dynamical behavior in parameter space.

Experimental work on the kicked rotor using a BoseEinstein condensate [1] has mostly concentrated on the short time behavior at quantum resonance or on the so-called antiresonance, where the motion is exactly periodic in the case $u=0$. But an experimental observation of the ballistic quantum resonance dynamics up to $10, \ldots, 30$ kicks seems possible
[28], for small enough kicking strength $k$ so as to avoid a too fast spread in momentum space which cannot be monitored by standard time-of-flight detection $[7,8]$. Our results are fully consistent with the few published experimental data, which show that both resonant and antiresonant dynamics essentially survive the presence of small nonlinearities, apart from other effects which damp, for instance, the periodic oscillations at the antiresonance. Such effects are, e.g., the uncertainty of the center of the initial momentum distribution, and fluctuations in the experimental kicking strength [1].

\section{ACKNOWLEDGMENTS}

L.R. and R.A. acknowledge partial support from the MIUR-PRIN project "Order and Chaos in Nonlinear Extended Systems: Coherent Structures, Weak Stochasticity and Anomalous Transport," and the INFM Advanced Project "Weak Chaos: Theory and Applications." S.W. thanks Professor Ken Taylor for his hospitality and financial support at the Queen's University of Belfast, where part of the present work originated. Enlightening discussions with Professor E. Arimondo and O. Morsch on the experimental possibilities and with R. Mannella on Lévy statistics are gratefully acknowledged.
[1] L. Deng et al., Phys. Rev. Lett. 83, 5407 (1999); G. Duffy et al., cond-mat/0401346; cond-mat/0406545.

[2] C. J. Pethick and H. Smith, Bose-Einstein Condensation in Dilute Gases, (Cambridge University Press, Cambridge, U.K., 2002).

[3] F. Dalfovo et al., Rev. Mod. Phys. 71, 463 (1999).

[4] C. Zhang et al., Phys. Rev. Lett. 92, 054101 (2004).

[5] S. A. Gardiner et al., Phys. Rev. A 62, 023612 (2000).

[6] F. L. Moore et al., Phys. Rev. Lett. 75, 4598 (1995); H. Ammann et al., ibid. 80, 4111 (1998); J. Ringot et al., ibid. 85, 2741 (2000); M. B. d'Arcy et al., ibid. 87, 074102 (2001); M. P. Sadgrove et al., e-print quant-ph/0401161.

[7] S. Wimberger, I. Guarneri, and S. Fishman, Nonlinearity 16, 1381 (2003).

[8] M. B. d'Arcy et al., Phys. Rev. E 69, 027201 (2004).

[9] D. A. Steck et al., Phys. Rev. E 62, 3461 (2000).

[10] F. Benvenuto et al., Phys. Rev. A 44, R3423 (1991).

[11] See, e.g., A. D. Bandrauk and H. Shen, J. Chem. Phys. 99, 1185 (1993).

[12] M. Reed and B. Simon, Functional Analysis (Academic Press, San Diego, 1980).

[13] D. Shepelyansky, Phys. Rev. Lett. 70, 1787 (1993).

[14] G. Casati et al., in Stochastic Behavior in Classical and Quan- tum Hamiltonian Systems edited by G. Casati and J. Ford (Springer-Verlag, Berlin, 1979), p. 334.

[15] F. M. Izrailev, Phys. Rep. 196, 299 (1990).

[16] B. V. Chirikov, in Chaos and Quantum Physics, edited by M. J. Giannoni, A. Voros, and J. Zinn-Justin (North-Holland, Amsterdam, 1991).

[17] F. M. Izrailev and D. L. Shepelyansky, Dokl. Akad. Nauk SSSR 249, 1103 (1979); Teor. Mat. Fiz. 43, 417 (1980).

[18] R. Artuso and L. Rebuzzini, Phys. Rev. E 66, 017203 (2002).

[19] See, e.g., also O. Morsch et al., Phys. Rev. Lett. 87, 140402 (2001).

[20] R. Artuso and L. Rebuzzini, Phys. Rev. E 68, 036221 (2003).

[21] T. Kottos and M. Weiss, Phys. Rev. Lett. 93, 190604 (2004).

[22] G. Casati et al., Phys. Rev. A 34,1413 (1986).

[23] L. Hufnagel et al., Phys. Rev. E 64, 012301 (2001).

[24] M. Stefancich et al., Phys. Rev. E 57, 6625 (1998).

[25] S. Wimberger, I. Guarneri, and S. Fishman, Phys. Rev. Lett. 92, 084102 (2004).

[26] S. Fishman, I. Guarneri, and L. Rebuzzini, J. Stat. Phys. 110, 911 (2003).

[27] G. L. Alfimov et al., Phys. Rev. E 66, 046608 (2002).

[28] S. Wimberger, R. Mannella, O. Morsch, and E. Arimondo, Phys. Rev. Lett. cond-mat/0501565. 